\title{
Preventing NETosis to reduce thrombosis
}

$c 4$

targeting the
extracellular
adenosine
signalling
pathway
can prevent
aPL-induced
NETosis

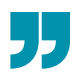

Although the pathogenesis of antiphospholipid syndrome (APS) is not fully understood, one important mechanism in thrombus formation is thought to be neutrophil extracellular trap (NET) formation (known as NETosis) induced by antiphospholipid antibodies (aPL). The results of a new study published in Nature Communications suggest that targeting the extracellular adenosine signalling pathway can prevent aPL-induced NETosis, thereby reducing clot formation in mouse models of thrombosis.

"The current approach to the treatment of APS with anticoagulants does not prevent all cases of blood clotting," explains corresponding author Jason Knight. "Furthermore, anticoagulants have very little effect on the neurologic, haematologic and cardiac complications that regularly affect patients with APS. Our group has been pursuing the hypothesis that anti-inflammatory treatment might provide a more targeted way to treat APS, giving better control of the condition with fewer adverse effects."

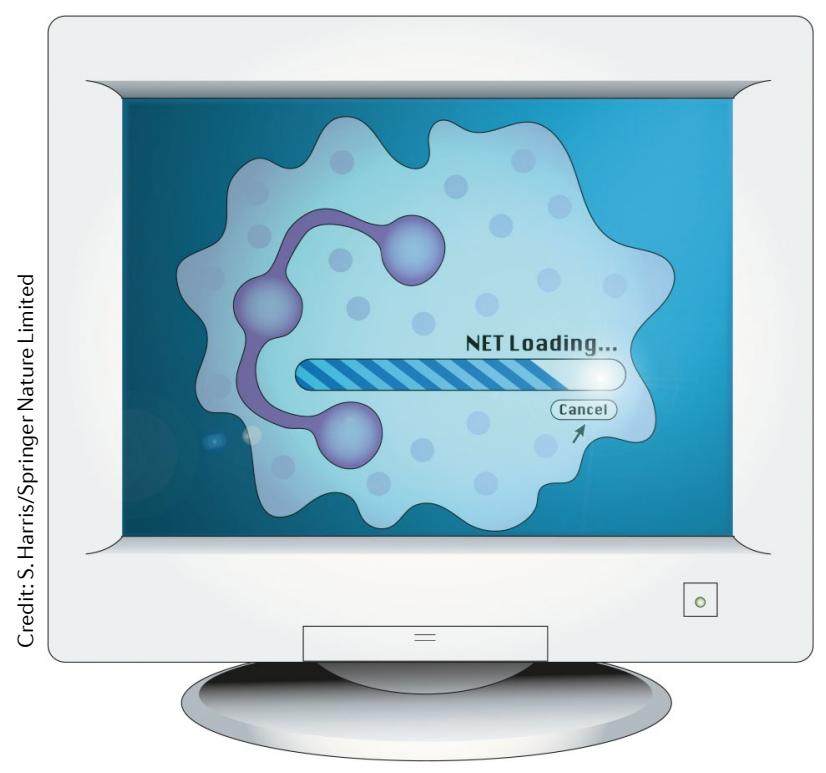

Building on previous work on the function of neutrophils and NETs in APS-related thrombosis, Knight and colleagues investigated a potential role for the adenosine signalling pathway in preventing NETosis. The activation of cell surface adenosine receptors regulates the production of the second messenger cyclic AMP (cAMP), which can suppress NET formation in certain circumstances. The researchers therefore hypothesized that stimulating these adenosine receptors with agonists might reduce NETosis and, hence, thrombosis.

Agonists for the adenosine receptors $A_{2 B}$ and $A_{3}$ did not affect NETosis, but the adenosine receptor $\mathrm{A}_{2 \mathrm{~A}}$ agonist CGS21680 prevented ex vivo aPL-induced NETosis in a cAMP-dependent manner in neutrophils from healthy individuals stimulated with either aPL from serum pooled from patients with APS or with purified anti- $\beta 2$-glycoprotein I antibodies.

"We found that exposure of neutrophils to CGS21680 very effectively prevented NET release," states Knight. "Activating adenosine receptors on the neutrophil surface seems to be a natural pathway for turning off inflammation, which we now show can be harnessed for the prevention of NET release."

The researchers next investigated the effects of CGS21680 in vivo using a mouse model of venous thrombosis in which blood flow is restricted by constriction of the vena cava. In this model, administration of CGS21680 reduced the number of NETs detectable in the plasma of the mice, as well as the incidence, size and weight of clots compared with untreated mice. The addition of exogenous aPL to this venous thrombosis model to create an APSlike model of thrombosis increased the size of the clots, an effect that was reversed by CGS21680.

"Interestingly, we found that activating adenosine receptors not only reduced blood clots in mice with APS, but also in healthy mice, suggesting that this pathway might also be applicable for conditions like deep vein thrombosis and pulmonary embolism in the general population," says Knight.

CGS21680 is not currently approved for use in humans, so the researchers tested dipyridamole, an antithrombotic medication that enhances the adenosine signalling pathway. Similar to CGS21680, dipyridamole reduced NET release and clot formation in mice with APS-like disease.

"From a clinical point of view, it is of interest that, like CGS21680, dipyridamole suppresses aPLmediated NETosis via the adenosine $\mathrm{A}_{2 \mathrm{~A}}$ receptor and mitigates venous thrombosis in mice," comments Ricard Cervera, who was not involved in this study. "Medications with similar adenosine-amplifying properties to dipyridamole have been reported to be effective in either case reports or preclinical models of APS. Therefore, a clinical trial to test the efficacy of dipyridamole in patients with this syndrome should be designed."

Although dipyridamole has been approved by the FDA for use in the prevention of post-operative thrombosis following cardiac valve replacement, it has never been tested in patients with APS. "Based on the findings in this study, we are now planning a pilot clinical trial in patients with APS," concludes Knight.

Joanna Collison

ORIGINAL ARTICLE Ali, R. A. et al. Adenosine receptor agonism protects against NETosis and thrombosis in antiphospholipid syndrome. Nat. Commun. 10, 1916 (2019) 